\title{
Homing in Pigeons: The Role of the Hippocampal Formation in the Representation of Landmarks Used for Navigation
}

\author{
Anna Gagliardo, ${ }^{1}$ Paolo loalé, ${ }^{1}$ and Verner P. Bingman ${ }^{2}$ \\ ${ }^{1}$ Dipartimento di Etologia, Ecologia ed Evoluzione, Universitá di Pisa, I-56126 Pisa, Italy, and ${ }^{2}$ Department of Psychology, \\ Bowling Green State University, Bowling Green, Ohio 43403
}

\begin{abstract}
When given repeated training from a location, homing pigeons acquire the ability to use familiar landmarks to navigate home. Both control and hippocampal-lesioned pigeons succeed in learning to use familiar landmarks for homing. However, the landmark representations that guide navigation are strikingly different. Control and hippocampal-lesioned pigeons were initially given repeated training flights from two locations. On subsequent test days from the two training locations, all pigeons were rendered anosmic to eliminate use of their navigational map and were phase- or clock-shifted to examine the extent to which their learned landmark representations were dependent on the use of the sun as a compass. We show that control pigeons acquire a landmark representation that allows
\end{abstract}

them to directly use landmarks without reference to the sun to guide their flight home, called "pilotage". Hippocampallesioned birds only learn to use familiar landmarks at the training location to recall the compass direction home, based on the sun, flown during training, called "site-specific compass orientation." The results demonstrate that for navigation of $20 \mathrm{~km}$ or more in a natural field setting, the hippocampal formation is necessary if homing pigeons are to learn a spatial representation based on numerous independent landmark elements that can be used to directly guide their return home.

Key words: cognitive map; hippocampus; homing; navigation; pigeons; spatial memory
The critical role of the amniote hippocampal formation (HF) in spatial cognition when map-like (O'Keefe and Nadel, 1978) or relational (Eichenbaum et al., 1994) landmark representations are used for navigation is a well described phenomenon. There is a considerable amount of data from rats (Morris et al., 1982; Eichenbaum et al., 1990) and birds (Sherry and Vaccarino, 1989; Strasser and Bingman 1997) demonstrating that hippocampal lesions impair or alter navigation by landmarks under laboratory conditions. The slower homing times of HF-lesioned homing pigeons (Columba livia) has also been used to suggest that the avian HF is critical for landmark navigation under natural conditions and distances of tens of kilometers (Bingman et al., 1988a, 1995; Bingman and Mench, 1990).

When homing pigeons are released from an unfamiliar location, they are limited to using their navigational map, together with some compass mechanism to navigate home (Kramer, 1959). However, when pigeons are released in a familiar area, they can also rely on a learned representation of familiar landmarks to navigate (Hartwick et al., 1977). (Familiar landmarks need not be exclusively visual but may include stimuli from any sensory channel.) HF-lesioned pigeons also succeed in learning to use familiar landmarks to navigate home (Bingman et al., 1988b).

Familiar landmark navigation is complicated by the independent ability of pigeons to use the sun as a compass ("sun compass") (Schmidt-Koenig, 1961). In fact, homing pigeons can use familiar landmarks for navigation relying on two distinct mecha-

\footnotetext{
Received July 16, 1998; revised Sept. 11, 1998; accepted Sept. 23, 1998.

This work was supported by a NATO collaborative research grant (V.P.B.) and grants from the National Institutes of Mental Health (V.P.B.) and Consiglio Nazionale delle Ricerche (P.I.). We thank Meliha Duncan for her help in preparing this manuscript.

Correspondence should be addressed to Verner P. Bingman, Department of Psychology, Bowling Green State University, Bowling Green, OH 43403. Copyright (C) 1998 Society for Neuroscience $0270-6474 / 98 / 190311-05 \$ 05.00 / 0$
}

nisms. First, a pigeon can navigate by directly referring to the landmarks experienced during training without referring to any compass mechanism, which we will call "pilotage" (Bingman and Ioalé, 1989). Alternatively, a pigeon can use the landmarks at a familiar location only to recall the compass (sun) direction home flown from that location, which we will call "site-specific compass orientation” (Luschi and Dall'Antonia, 1993). These two strategies are strikingly different in the amount of information that would be extracted from the landmarks. For pilotage, familiar landmarks are used to form a spatial representation that directly guides navigation and is likely based on pigeons learning the spatial relationship among numerous independent landmark elements, including the home loft. For site-specific compass orientation, local landmarks in the immediate vicinity of a training location are used only to recall a compass direction, and it is the sun compass that actually directs the flight home.

Both intact and HF-lesioned pigeons succeed in learning to use familiar landmarks for navigation. However, in the present paper, we show that the landmark representations that guide their navigation are strikingly different. Control pigeons learn a spatial representation that allows them to directly use landmarks to guide their flight home: pilotage. Hippocampal-lesioned birds, in contrast, learn to use familiar landmarks only to recall the compass direction home: site-specific compass orientation.

\section{MATERIALS AND METHODS}

Subjects. All pigeons were housed near Pisa, Italy. Two independent series of pigeons were used. In series 1,28 (16 controls and $12 \mathrm{HF}$ lesioned) pigeons with previous homing experience limited to free flights around the loft were used. Surgery (see below) took place $\sim 6$ months before experimental training. In series 2, 44 (27 controls and $17 \mathrm{HF}$ lesioned) pigeons with previous homing experience limited to free flights around the loft were used. Surgery took place $\sim 1$ month before experimental training.

Surgery. Aspiration procedures were used to lesion the HF, consisting 
of a medial hippocampus and dorsomedial parahippocampus (Karten and Hodos, 1967), of the lesioned birds. The methods have been described in detail elsewhere (Bingman et al., 1984). With aspiration lesions, there is always the risk that any behavioral effects could be caused by damage of fibers of passage. However, in birds, the only fibers that pass through HF that do not originate or terminate there is a projection from the hyperstriatum accessorium to the midbrain (Reiner and Karten, 1983). Aspiration lesions to the hyperstriatum accessorium have been repeatedly used as control lesions (Bingman et al., 1984, 1988a), and no effect on homing behavior has ever been observed after damage to this area. Therefore, it is unlikely that any observed effects could be caused by damage to fibers of passage.

Training from familiar locations. Before the experimental releases, the pigeons from both series were given eight training releases under sunny conditions from each of two locations: Livorno, $13.6 \mathrm{~km}$ southeast of home; and La Costanza, $16.7 \mathrm{~km}$ north of home. The site of a training release switched randomly between Livorno and La Costanza with the constraint that the same training location was not used for 3 consecutive days. During the first seven training releases, the pigeons were released together as a flock. During the last training release, the pigeons were released individually, and vanishing bearings were recorded.

Experimental releases: series 1. After training, the pigeons were subjected to two experimental releases, one from each training location. The two landmark-based navigational mechanisms described above differ primarily in what guides the orientation of a pigeon; for pilotage, a pigeon would refer to the landmarks themselves, whereas for site-specific compass orientation, a pigeon, on sunny days, would orient by its sun compass. Therefore, to determine which of these mechanisms a pigeon was using, all pigeons before each experimental release were phase- or clock-shifted $6 \mathrm{hr}$ fast for at least $7 \mathrm{~d}$. Phase-shifting birds $6 \mathrm{hr}$ fast leads to a counter-clockwise shift in orientation compared with nonshifted birds when the sun compass is used for orientation. Because the present study was performed in late spring and summer, a $6 \mathrm{hr}$ fast shift would cause a shift in orientation of $\sim 120^{\circ}$ if the sun compass was being used exclusively for orientation. The orientation of pigeons relying exclusively on a pilotage mechanism should not be affected by a phase-shift manipulation.

To examine landmark navigation, it was also necessary to eliminate the possibility that the experimental pigeons could rely on their navigational map to determine their location relative to home (Kramer, 1959). It has been overwhelming demonstrated that for pigeons in Italy atmospheric odors are the crucial environmental stimuli used in the operation of the navigational map (Papi, 1990). Therefore, to eliminate the possibility that the pigeons would use their navigational map for homing, all the birds were rendered anosmic by intranasal injection of $4 \%$ zinc sulfate (2 $\mathrm{ml}$ in each nostril) $24 \mathrm{hr}$ before each release (Benvenuti et al., 1992). In summary, for the experimental releases, the anosmic HF-lesioned and control pigeons were unable to use their navigational map to home and were therefore limited to relying on the landmark representation acquired during training. Further, the same pigeons were phase-shifted $6 \mathrm{hr}$ fast, which permits dissociation of a pilotage landmark mechanism from a site-specific compass orientation mechanism based on the sun.

Series 1 birds were first released from La Costanza. Returning birds were phase-shifted again for $7 \mathrm{~d}$ and then released from Livorno. Birds were released singly and followed with $10 \times 40$ binoculars until they disappeared from view. The vanishing bearing and vanishing time of a bird were recorded, and then the next bird was released, alternating treatment groups. An observer at the loft recorded the arrival of the pigeons so that homing times could be calculated. Circular statistics were used to examine the distribution of vanishing bearings for each group for each experimental release (Batschelet, 1981). The Rayleigh test was used to determine whether the vanishing bearings of a group deviated from uniform. The Watson-Williams $U^{2}$ test was used for between-group differences in vanishing bearings, and the Mann-Whitney $U$ test was used for differences in homing times.

Experimental releases: series 2. Identical to series 1, the series 2 pigeons consisted of a group of control $(n=14)$ and a group of HF-lesioned $(n=$ 17) birds that were both phase-shifted and rendered anosmic before the experimental releases (see above). Two HF-lesioned birds only participated in the second release from Livorno. In addition, a second group of controls $(n=13)$, not rendered anosmic and therefore able to use their navigational map based on atmospheric odors, was included to determine whether the behavioral differences observed in series 1 was a consequence of the two groups responding differently to the phase-shift manipulation (see below).
Histology. After completion of the experimental releases, 10 remaining HF-lesioned pigeons from series 1 and series 2 were killed for histology and lesion reconstruction using procedures described elsewhere (Bingman et al., 1984). Because many HF-lesioned birds were lost, there is a danger that the remaining birds do not adequately represent the lesion damage experienced by the group as a whole. However, the behavior (orientation) of the remaining pigeons was indistinguishable from the birds lost. Also, we have conducted numerous experiments with HFlesioned pigeons (Bingman et al., 1995), and the surgical procedures have been standardized such that there is very little variability in the extent of lesion damage among birds. The avian HF is visible while aspirating, thus permitting excellent control during lesion surgery. Consequently, we are confident that the sampled pigeons offer a reasonable approximation of the lesion damage sustained by the HF-lesioned birds as a whole.

\section{RESULTS \\ Histology}

Summarized in Figure 1 is the lesion damage observed in the sampled pigeons. All birds sustained considerable bilateral damage to the hippocampus, and most sustained considerable bilateral damage to the parahippocampus. Occasional damage to neighboring hyperstriatum accessorium, hyperstriatum ventrale, and neostriatum was also observed. On average, pigeons sustained $77 \%(\mathrm{SE}=2.0)$ bilateral damage to the hippocampus and $69 \%(\mathrm{SE}=2.9)$ damage to the parahippocampus. No one bird sustained $>10 \%$, and no more than four birds sustained $>5 \%$ bilateral damage to any other structure. In general, the lesion damage resembles closely that observed in other aspiration lesion studies (Bingman et al., 1988a,b).

\section{Behavior}

Summarized in Figure 2 and Table 1 are the data and statistical analyses from the experimental releases from the two series. It should be noted that on the last training release from both sites, the control and HF-lesioned birds were impressively and indistinguishably well oriented toward home (Table 1). Examining the initial orientation recorded during the experimental releases, the phase-shifted, anosmic control, and HF-lesioned birds were strikingly different. The vanishing bearings of the control birds, although shifted slightly counter-clockwise in the expected phaseshift direction, were nonetheless close to the homeward direction. This result demonstrates that their orientation was primarily guided by familiar landmarks and not the sun compass. In contrast, the vanishing bearings of the HF-lesioned birds showed the typical large counter-clockwise displacement from the home direction characteristic of fast phase-shift treatments, demonstrating primary use of the sun compass for orientation. The control birds also displayed consistently faster median homing times.

The data from the phase-shifted anosmic pigeons is entirely consistent with the hypothesis that although HF-lesioned birds can learn to use familiar landmarks to orient home (Bingman et al., 1988b), the structure of their landmark representation is very different from that learned by controls. However, previous research has shown that when control and HF-lesioned birds are phase-shifted and then released from a distant unfamiliar location (no familiar landmarks available) with their ability to smell intact, control birds often show a smaller shift in orientation compared with HF-lesioned birds (Bingman et al., 1996). The difference in orientation under these conditions is generally only $\sim 30^{\circ}$, considerably smaller than the $100^{\circ}$ or larger differences observed in the present study. Nonetheless, it was important to determine whether the observed differences in orientation were indeed attributable to differences in the learned landmark representations rather than a general difference in how intact and HFlesioned birds respond to phase-shifting. 


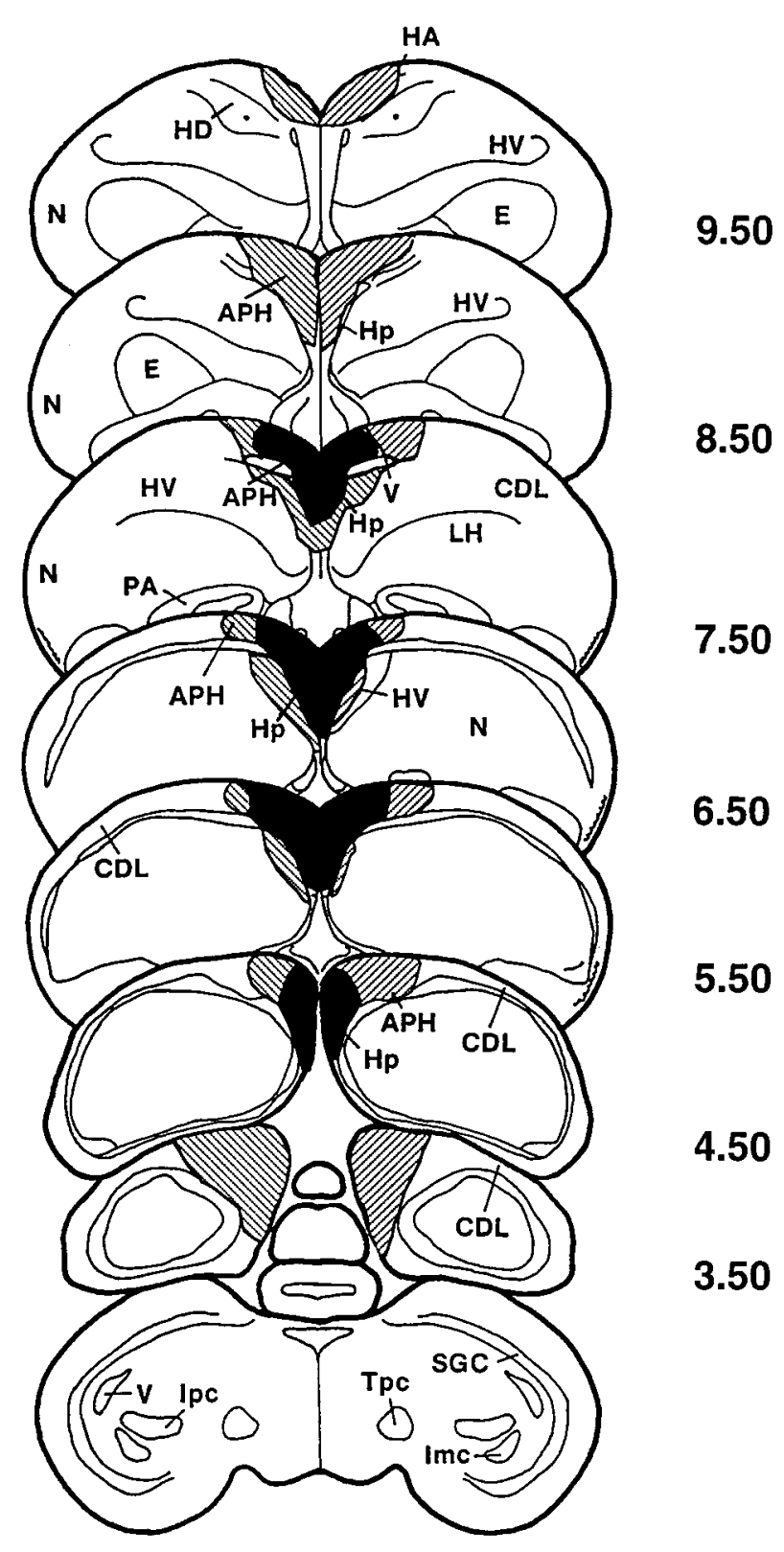

Figure 1. Lesion reconstruction summary of the 10 sampled pigeons. Dark areas indicate lesion damage found in at least 8 of the 10 pigeons. Striped areas indicate lesion damage in at least 3 of the 10 pigeons. $A P H$, Parahippocampus; $C D L$, area corticoidea dorsolateralis; $E$, ectostriatum; $H A$, hyperstriatum accessorium; $H D$, hyperstriatum dorsale; $H p$, hippocampus; $H V$, hyperstriatum ventrale; Imc, nucleus isthmi, pars magnocellularis; Ipc, nucleus isthmi, pars parvocellularis; $L H$, lamina hyperstriatica; $N$, neostriatum; $P A$, paleostriatum augmentatum; $S G C$, stratum griseum centrale; $T p c$, nucleus tegmenti peduncolo-pontinus, pars compacta; $V$, ventriculus. Adapted from the atlas of Karten and Hodos (1967).

Birds that can use both familiar landmarks and a navigational map to determine their location relative to home rely primarily on their navigational map, together with sun compass for navigation (Füller et al., 1983; Bingman et al., 1989). Therefore, the series 2 control birds that were able to smell (navigational map intact) were expected to show a qualitatively larger counterclockwise shift in orientation compared with anosmic controls. Indeed, an examination of Figure 2 and Table 1 (series 2 only) reveals that the shift in vanishing bearings away from the home- ward direction was larger for the controls that were able to smell compared with the anosmic controls, although a significant difference was only found from Livorno. It is also worth noting that no statistical differences were found in the vanishing bearings of the anosmic HF-lesioned birds and controls able to smell. Therefore, the large difference in vanishing bearings between the anosmic controls and anosmic HF-lesioned birds was not a simple consequence of the groups responding differently to a phase-shift manipulation.

\section{DISCUSSION}

It has been shown previously that both control and HF-lesioned birds can rely on familiar landmarks from a training site to navigate home when their navigational map based on atmospheric odors is rendered dysfunctional (Bingman et al., 1988b). The present results, however, demonstrate that the structure of the landmark representation used to guide navigation is fundamentally different between the two groups. The control birds were generally insensitive to the phase-shift manipulation, displaying homeward orientation comparable to the last training releases. This behavior is consistent with the learning of a landmark representation that can directly guide the in-flight orientation of a pigeon (pilotage) without reference to an independent sun compass mechanism (Bingman and Ioalé, 1989). Their modest shift in orientation in the expected phase-shift direction, however, suggests that in addition to landmark-based pilotage, controls also learned a site-specific compass orientation response that influenced their behavior.

The behavior of the HF-lesioned birds could not be more strikingly different. They were as well oriented as controls (similar mean vector lengths) but showed a dramatic counter-clockwise shift in orientation, demonstrating that they were relying exclusively on their sun compass to guide their in-flight orientation. In contrast to controls, they used familiar landmarks only to recall a site-specific compass orientation response based on the sun compass (Luschi and Dall'Antonia, 1993).

In our opinion, the data presented provide the first unambiguous evidence demonstrating the critical role of the amniote hippocampal formation in landmark navigational learning in a natural field setting. It is important to note that the landmark representation acquired by the control birds was not a simple chaining of responses acquired during training, i.e., fly to the barn, then the water tower, then the power lines, etc. The vanishing bearings of the control birds were shifted slightly compared with the last training releases, indicating that they were not simply following the same path taken during training and needed to make route adjustments during the flight home. Also, pigeons are known to successfully rely on familiar landmarks for navigation, even when they are released several kilometers from a training release site (Bingman et al., 1989). Although speculative, the characteristics of the landmark representation acquired by the control birds suggests that the learned representation captured the spatial relationship among numerous landmarks found between the release site and the home loft: a kind of relational representation (Eichenbaum et al., 1994) with map-like properties (O'Keefe and Nadel, 1978).

It is clear that the pigeons with HF lesions failed to acquire a similarly rich landmark representation. The birds did learn, and what they learned would have gotten them home quickly if they were not phase-shifted (Bingman et al., 1988b). However, the acquired landmark representation of the HF-lesioned birds served only to recall an associated sun compass direction. The 
Figure 2. Vanishing bearings of anosmic controls (filled circles), anosmic hippocampal-lesioned birds (striped triangles), and control birds able to smell (open circles) recorded during the experimental releases from the familiar training locations. All pigeons were phaseshifted. Each data point on the periphery of a circle represents the vanishing bearing of one bird. Arrows in each circle represent the direction of the mean vector for each group. The length of the arrow approximates the mean vector length for a group, with the radius of the circle equal to 1.0 .

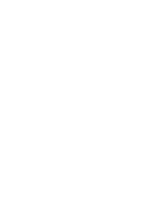

Series 1
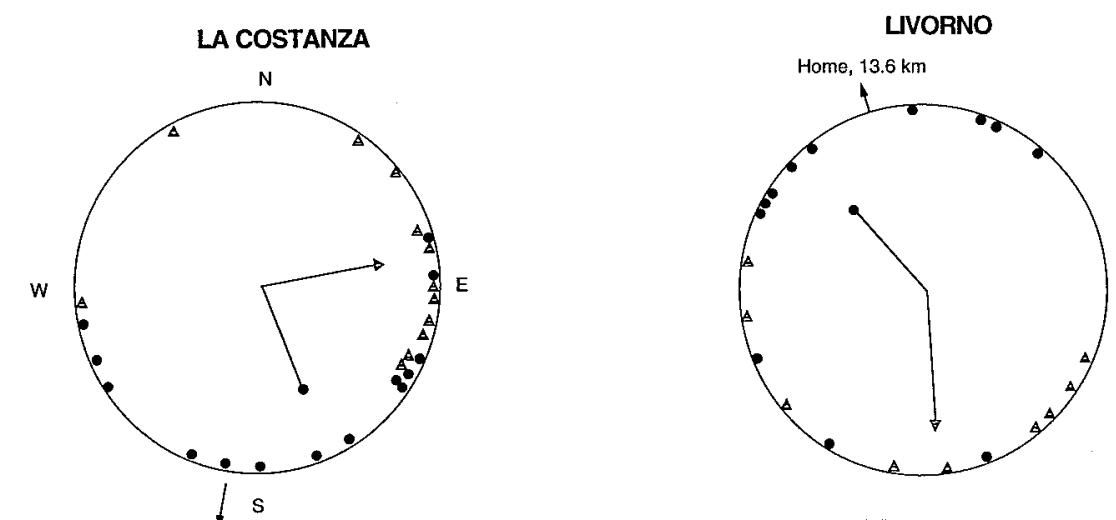

Home, $16.7 \mathrm{~km}$

- ANOSMIC CONTROLS

A ANOSMIC, HIPPOCAMPAL LESIONED EXPERIMENTALS

- CONTROLS ABLE TO SMELL

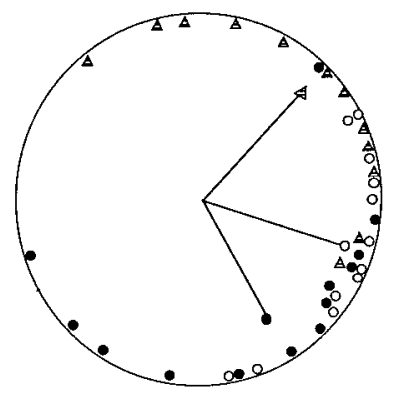

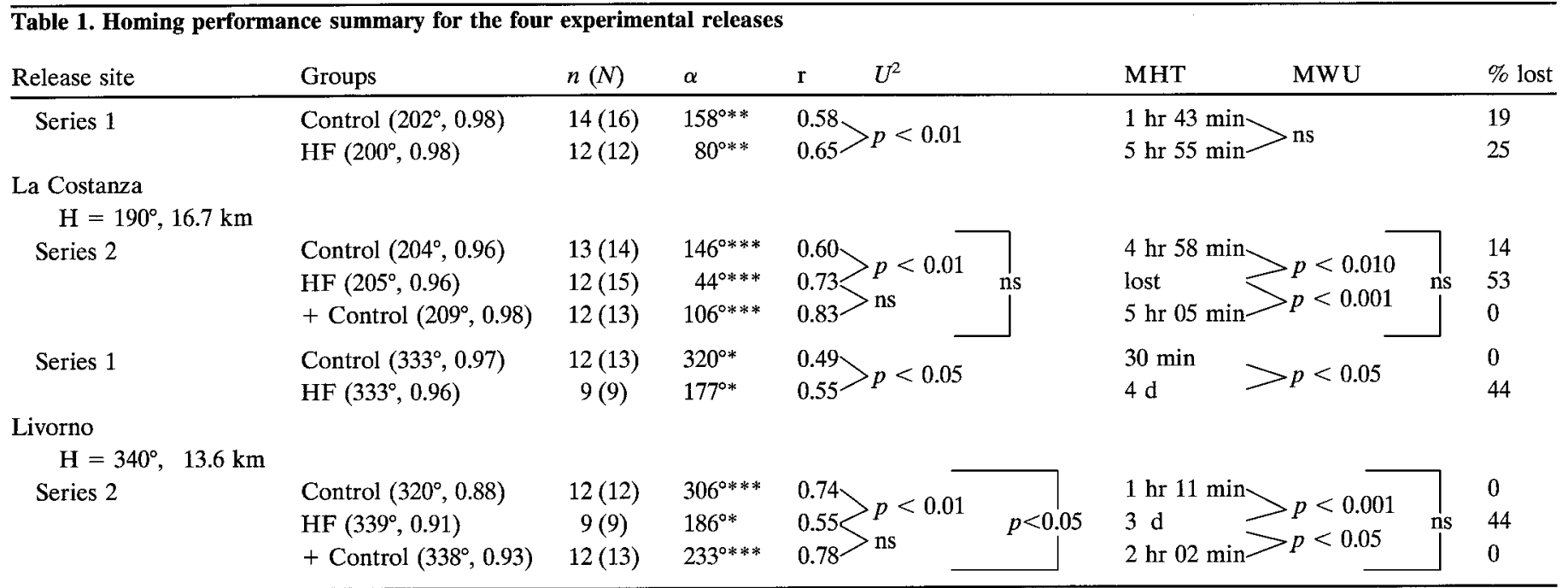

Groups: Control, anosmic controls; HF, hippocampal lesioned anosmic; + Control, controls able to smell. All pigeons were phase-shifted; values in parentheses identify the group mean vanishing bearing and mean vector length from the last training release. $n$, Number of vanishing bearings recorded; $N$, number of birds released. $\alpha$, Mean vanishing bearing; ${ }^{*} p \leq 0.05 ;{ }^{* *} p \leq 0.01 ;{ }^{* * *} p \leq 0.001$ with a Rayleigh test. r, Mean vector length. $U^{2}$, Beiween-group differences tested with the Watson-Williams $U^{2}$ test. MHT, Median homing time; lost, less than half the birds returned. MWU, Between-group differences in median homing time tested with a Mann-Whitney $U$ test. \% lost, Percent of birds that failed to return. ns, Not significant.

deficiency of such an impoverished spatial representation is highlighted by the considerably longer homing times and the large number of HF-lesioned birds that never returned home (Table 1). It is clear that the landmark representation acquired by the HF-lesioned birds did not allow them to readily correct for the errors in initial orientation created by the phase-shift manipulation. In summary, they did not acquire a landmark representation that would have permitted pilotage.

One curious finding is the slight difference in the behavior of the control birds from Livorno and La Costanza. The data suggest that the control birds were more likely to exclusively use a pilotage strategy from Livorno than from La Costanza. If real, what might be the origin of this difference? One possibility is that different locations will vary in the richness and complexity of landmarks, and this may influence the extent to which pilotage or site-specific compass orientation will be used. The Livorno training site was near a large urban center with heavy industrial landmarks visible for a considerable distance. The La Costanza 
training site was in a more uniform agricultural area. The difference in the type of landmarks at the two locations may explain why pilotage was more apparent from Livorno.

Finally, it has been reported previously that when tested in an experimental arena HF-lesioned homing pigeons are unable to use their sun compass to locate a food goal (Bingman and Jones, 1994). The success of the HF-lesioned birds in the present study to learn a sun compass-based site-specific orientation response from a familiar training location was therefore unexpected. In the arena study, the HF-lesioned pigeons were limited to using their sun compass to learn the direction of a goal while being held within an enclosed space. In contrast, during training flights in the present study, HF-lesioned pigeons were able to fly, use their navigational map, experience landmarks, and orient by their sun compass. One can therefore reconcile the apparently contradictory results of the present study and the previous arena study by recognizing that the arena study took place in a restricted stimulus-poor environment, whereas in the present study, learning took place in a stimulus-rich natural setting that allowed the expression of a broad range of behaviors. The results of the present study highlight the unique importance of performing experiments under natural conditions in attempting to unravel the complex relationship among brain, behavior, and cognition. It would have been impossible to reveal the fundamental difference in the landmark representations of control and HF-lesioned birds in a laboratory setting. The relationship between hippocampus and spatial cognition evolved under natural conditions, and experiments performed under natural or semi-natural conditions are a critical source of new perspectives needed for a more thorough and perhaps more challenging understanding of the role of $\mathrm{HF}$ in cognition.

\section{REFERENCES}

Batschelet E (1981) Circular statistics in biology. New York: Academic. Benvenuti S, Ioalé P, Gagliardo A, Bonadonna F (1992) Effects of zinc sulfate-induced anosmia on homing behaviour of pigeons. Comp Biochem Physiol 103A:519-526.

Bingman VP, Ioalé P (1989) Initial orientation of homing pigeons based on information gathered at familiar release sites remains homeward directed following clock-shift. Behaviour 110:205-218.

Bingman VP, Jones T-J (1994) Sun compass-based spatial learning impaired in homing pigeons with hippocampal lesions. J Neurosci 14:6687-6694.

Bingman VP, Mench JA (1990) Homing behavior of hippocampus and parahippocampus lesioned pigeons following short-distance releases. Behav Brain Res 40:227-238.
Bingman VP, Bagnoli P, Ioalé P, Casini G (1984) Homing behavior of pigeons after telencephalic ablations. Brain Behav Evol 24:94-106.

Bingman VP, Ioalé P, Casini G, Bagnoli P (1988a) Hippocampal ablated homing pigeons show a persistent impairment in the time taken to return home. J Comp Physiol [A] 163:559-563.

Bingman VP, Ioalé P, Casini G, Bagnoli P (1988b) Unimpaired acquisition of spatial reference memory but impaired homing performance in pigeons following hippocampal ablation. Behav Brain Res 27:179-187.

Bingman VP, Bagnoli P, Ioalé P, Casini G (1989) Behavioral and anatomical studies of the avian hippocampus. In: The hippocampus: new vistas, Vol 52, Neurology and neurobiology (Chan-Palay V, Koehler C, eds), pp 379-394. New York: Liss.

Bingman VP, Jones T-J, Strasser R, Gagliardo A, Ioalé P (1995) Homing pigeons, hippocampus and spatial cognition. In: Behavioural brain research in naturalistic and semi-naturalistic settings (Alleva E, Fasolo A, Lipp H-P, Nadel L, Ricceri L, eds), pp 207-223. Dordrecht, The Netherlands: Kluwer Academic.

Bingman VP, Gagliardo A, Ioalé P (1996) Hippocampal participation in the sun compass orientation of phase-shifted homing pigeons. J Comp Physiol [A] 179:695-702.

Eichenbaum H, Stewart C, Morris RGM (1990) Hippocampal representation in place learning. J Neurosci 10:2531-2542.

Eichenbaum H, Otto T, Cohen NJ (1994) Two functional components of the hippocampal memory system. Behav Brain Sci 17:449-517.

Füller E, Kowalski U, Wiltschko R (1983) Orientation of homing pigeons: compass orientation or piloting by landmarks. J Comp Physiol 153:55-58.

Hartwick RF, Foá A, Papi F (1977) The effect of olfactory deprivation by nasal tubes upon homing behaviour in pigeons. Behav Ecol Sociobiol 2:81-89.

Karten HJ, Hodos W (1967) A stereotaxic atlas of the brain of the pigeons (Columba livia). Baltimore: Johns Hopkins UP.

Kramer G (1959) Recent experiments on bird orientation. Ibis 101:399-416.

Luschi P, Dall'Antonia P (1993) Anosmic pigeons orient from familiar sites by relying on the map-and-compass mechanism. Anim Behav 46:1195-1203.

Morris RGM, Garrud P, Rawlins JNP, O'Keefe J (1982) Place navigation impaired in rats with hippocampal lesions. Nature 297:681-683.

O'Keefe J, Nadel L (1978) The hippocampus as a cognitive map. New York: Oxford UP.

Papi F (1990) Olfactory navigation in birds. Experientia 46:352-363.

Reiner A, Karten HJ (1983) The laminar source of efferent projections from the avian Wulst. Brain Res 275:349-354.

Schmidt-Koenig K (1961) Die Sonne als Kompass im Heimorientierungssystem der Brieftauben. Z Tierpsychol 68:221-224.

Sherry DF, Vaccarino AL (1989) Hippocampus and memory for food caches in black-capped chickadees. Behav Neurosci 103:308-318.

Strasser R, Bingman VP (1997) Goal recognition and hippocampal formation in the homing pigeon (Columba livia). Behav Neurosci 111: 1245-1256. 